\title{
MARCo de ANÁliSiS DEL SISTEMA DE PENSIONES CHILENO DESPUÉS DE LA REFORMA DE 2008*
}

\section{Pablo Arellano OrTiz ${ }^{* *}$}

RESUMEN: Desde el 1980 el régimen de fondos de pensión ha llamado la atención de los expertos en seguridad social en América Latina, pero también en Europa del Este. La reciente reforma de 2008, que extendió la cobertura de pensiones, fuerza a replantear el análisis del modelo chileno desde una perspectiva diferente. Mientras la protección del riesgo de la vejez es realizada sobre la base de un modelo de una sola lógica basado en capacidad contributiva, la conformidad del derecho a pensión con los principios de universalidad subjetiva debe ser analizada. Los dos componentes del sistema chileno deberían ser entendidos para poder valorar su capacidad de cubrir a toda la población chilena. El primero de los componentes incluye un mecanismo no contributivo suministrado por el Estado, que responde a sus obligaciones internacionales y constitucionales de protección de su población. El segundo componente consiste en mecanismos contributivos obligatorios y voluntarios que permiten a la población obtener protección contra los riesgos de la vejez. El complemento de estos dos mecanismos es lo que confiere al derecho de pensión su carácter universal. El sistema de retiro se ha convertido en un todo coherente, combinando universalismo e individualismo para proteger a toda la población a través de un sistema que tiene una lógica única.

PALABRAS CLAVE: Seguridad social - sistema de pensiones prestaciones contributivas prestaciones no contributivas universalismo individualismo.

Fecha recepción: 18 de enero de 2012.

Fecha aceptación: 2 de octubre de 2012.

** Profesor de Derecho del Trabajo y Seguridad Social de la Universidad Austral de Chile. Doctor en Derecho Privado y Ciencias Criminales, Université de París Ouest La Défense Nanterre. Email: pablo.arellano@uach.cl 


\section{ANALYSIS FRAMEWORK OF THE CHILEAN PENSION SYSTEM AFTER THE 2008 REFORM}

ABSTRACT: Since 1980 the pension funds system has called the attention of social security experts from Latin America, as well as Eastern Europe. The recent reform from 2008, that broadens the coverage of pensions, forces to rethink the Chilean model from a different perspective. While protection to the risk of growing old is done based on a sole logic model, based as well on tax related benefits, conformity to the pension law with principles of subjective universality must be analyzed. Both components of the Chilean system should be understood in order to appraise its coverage capacity to all the Chilean population. The first component includes a non-tax related mechanism provided by the State, responding to its international and constitutional obligations of protection to the population. The second component consists of mandatory and voluntary tax-related mechanisms that allow the population to obtain protection for aging. The complement to both of these mechanisms is what grants pension law its universal character. The retirement system has become a coherent whole, combining Universalism and Individualism to protect all of the population through a system with a sole logic.

KEY WORDS: Social security - pension system tax related benefits non-tax related benefits Universalism Individualism.

Sumario: 1) Introducción. 2) Desde un sistema multipilar a una sola lógica de cobertura. 3) Universalismo. (3.1.) La pensión básica solidaria. (3.2.) El aporte solidario. 4) Conclusiones.

\section{1) INTRODUCCIÓN}

En el año 1980 la atención se tornó hacia Chile; la reforma de su sistema de pensión se presentó como una alternativa de solución a los problemas de envejecimiento de la población mundial. Hoy, la reciente integración de un denominado "pilar solidario" en el sistema de pensiones en Chile capturó de nuevo la atención del modelo de protección basado en ahorros individuales. Pese a que la reforma de $2008^{1}$ no es la primera re-

Ley $\mathrm{n}^{\circ} 20.255$, D.O. 17 de marzo de 2008. Acerca de los diferentes aspectos de esta reforma ver: Cifuentes Lillo, Hugo (2008a) Reforma PREVISiOnal. Santiago: LexisNexis; Walker E., Francisco y Cifuentes Lillo, Hugo (2008) "Aspectos generales de la ley que 
forma ${ }^{2}$ al modelo chileno, y si hablamos de un sistema en constante evolución, esta reforma es indudablemente una de las importantes en las que las metas son la extensión de la cobertura de los beneficios para la vejez.

En 1980, Chile fue el primer país en adoptar un sistema de pensión estableciendo fondos de pensiones como un régimen básico obligatorio para proteger el riesgo de la vejez. Esta concepción de seguridad social ha sido seguida por una ola de reformas, llamadas de segunda generación,

establece una reforma previsional". Revista Laboral Chilena, abril, pp. 88-127 ; Cifuentes Lillo, Hugo (2008b) "Comentario sobre la reforma previsional chilena de 2008". Revista de la red de expertos iberoamericanos en seguridad social, Ceddet, OISS, No 2, primer semestre de 2008, pp. 8-12.

2 D.L. No 3.626 D.O. 21/02/81; D.L. No 3.650 D.O. 11/03/81; Ley No 18.072 D.O. 01/12/81; Ley No 18.086 D.O. 31/12/81; Ley No 18.137 D.O. 05/07/82; Ley No 18.196 D.O. 29/12/82; Ley No 18.208 D.O. 26/01/83; Ley No 18.225 D.O. 28/06/83; Ley No 18.379 D.O. $04 / 01 / 85$; Ley No 18.398 D.O. 24/01/85; Ley No 18.420 D.O. $05 / 07 / 85$; Ley No 18.481 D.O. 24/12/85; Ley No 18.482 D.O. $28 / 12 / 85$; Ley No 18.520 D.O. 13/06/86; Ley No 18.646 D.O. 29/08/87; Ley No 18.681 D.O. 31/12/87; Ley No 18.717 D.O. 28/05/88; Ley No 18.753 D.O. 28/10/88; Ley No 18.768 D.O. 29/12/88; Ley No 18.798 D.O. 23/05/89; Ley No 18.840 D.O. 10/10/89; Ley No 18.964 D.O. 10/03/90; Ley No 19.177 D.O. 27/11/92; Ley No 19.200 D.O. 18/01/93; Ley No 19.247 D.O. 15/09/93; Ley No 19.260 D.O. 04/12/93; Ley No 19.301 D.O. 19/03/94; Ley No 19.350 D.O. 14/11/94; Ley No 19.389 D.O. 18/05/95; Ley No 19.398 D.O. 04/08/95; Ley No 19.404 D.O. 21/08/95; Ley No 19.415 D.O. 30/09/95; Ley No 19.469 D.O. 03/09/96; Ley No 19.601 D.O. 18/01/99; Ley No 19.641 D.O. 28/10/99; Ley No 19.705 D.O. 20/12/00; Ley No 19.768 D.O. $07 / 11 / 01$; Ley No 19.795 D.O. 28/02/02; Ley No 19.895 D.O. 28/08/03; Ley No 19.934 D.O. 21/02/04; Ley No 20.023 D.O. 31/05/05; Ley No 20.190 D.O. $05 / 06 / 07$; Ley No 20.210 D.O. 31/07/07; Ley No 20.255 D.O. 17/03/08; Ley N 20.366 D.O. 29/07/09; Ley N ${ }^{\circ} 20.345$ D.O. 06/06/09; Ley Nº 20.448 D.O. 13/08/10; Ley Nº 20.455 D.O. 31/07/10. 


\section{que esparcieron este modelo hacia muchos países de América Latina ${ }^{3}$ y luego a Europa Oriental ${ }^{4}$. \\ Sin embargo, el modelo chileno ha causado muchos problemas de cobertura en Chile así como en otros países que han adoptado este me-}

Acerca de esta reforma en América Latina ver: CEPALC (2006) La protección social de cara al futuro: acceso, financiamiento y solidaridad. CEPALC, Naciones Unidas, Santiago; Matijascic, Milko y KaY, Stephen J. (2006) «La sécurité sociale à un carrefour: vers une réforme effective des pensions en Amérique Latine». Revue internationale de sécurité sociale, vol. 59, $\mathrm{N}^{\circ}$ 1, p. 3; Utноғғ, Andras (2006) «Brechas del Estado de bienestar y reformas de pensiones en América Latina", Revista de la CEPAL 89, agosto, p. 9; Gill, Indermit; Packard, Truman; Pugatch, Todd y Yermo, Juan (2005) «Repenser la sécurité sociale en Amérique latine». Revue internationale de sécurité sociale, vol. 58, $\mathrm{N}^{\circ}$ 2/3, p. 81; Tielman, Daniel y Uthoff, Andras (2005) «Assurance et protection sociale en Amérique latine», Revue internationale de sécurité sociale, vol. 58, $N^{\circ} 2 / 3$, p. 49; Mesa-Lago, Carmelo (2005) «Les réformes de la sécurité sociale en Amerique latine: observations sur le rapport de 2005 de la Banque Mondiale». Revue internationale de sécurité sociale, vol. 58 No. 2-3, p. 115; Ochando Claramunt, Carlos (2004) «Evaluation des réformes des systèmes de pensions latino-américaines», Revue internationale de sécurité sociale, vol. 57, $\mathrm{N}^{\circ}$ 2, p. 33; Bertranou, Fabio, Rofman, Rafael y Grushia, Carlos (2003) «De la réforme à la crise: le système de pensions en Argentine», Revue internationale de sécurité sociale, vol. 56, No2, p. 129; Muller, Katharina (2003) «The making of pension privatisation in Latin America and Eastern Europe». En Holzmann, Robert; Oreinstein, Mitchel y Rutkowki, Michal (eds.) (2003) Pension reform in Europe: Process and Progress, Directions in Development, The Wold Bank, Washington, pp. 47-78; Bertranous, Fabio y Rofman, Rafael (2002) «La prestation de sécurité sociale dans un contexte en plein évolution: l'expérience et les défis de l'Amérique latine». Revue internationale de sécurité sociale, vol. 55, $\mathrm{N}^{\circ} 1$, p. 75; Bonilla, Alejandro y ConTE-Grand, Alfredo (eds.) (1998) Pensiones en América Latina. Dos décadas de reforma, OIT, Ginebra, y de los mismos autores (1999) «Reforma de pensiones en América latina : desafíos pendientes, estrategias para el futuro». Análisis laboral, Marzo, p. 10; Lancereau, Thomas (1999) «Les fonds de pension en Amérique latine. Les fonds de pension latino-américains produiront-ils les effets que l'on prête au modèle chilien?» Futuribles, juillet - août, p. 109; VALDÉs-Prieto, Salvador (1997) «La sécurité sociale dans les réformes des retraites en Amérique latine». Economie Internationale, n ${ }^{\circ}$ 72, $4^{\circ}$ trimestre, p. 107; Gumucio, Juan Sebastián (1977) «Chile en el Mercosur: armonización de sistemas de seguridad social». Revista Laboral Chilena, Enero 1977, pp. 58-64; QueIsSER, Monika (1995) «Apres le Chili, les reformes de la deuxième génération en Amérique Latine». Revue internationale de sécurité sociale, vol. 48, 3/4, p. 27.

4 Acerca de esta reforma en Europa Oriental ver: OIT (2006) Pension reform in the Baltic States. Estonia - Lativia - Lithuania, Subregional Office for Central and Eastern Europe, Budapest, ILO; OIT (2005) Reforma de las pensiones en Europa central y oriental, Oficina subregional para Europa Central y Oriental, BIT, Budapest; FIAP (2004). La reforma de pensiones en Europa del Este: Experiencias y Perspectivas. Fiap, CIEDESS; Tortuero Plaza, José luis y Del Aguila Cazorla, Olimpia (2004) "Los sistemas de pensiones en Europa y Latinoamérica: realidades, expectativas e ideas para un debate". Revista del Ministerio de Trabajo y Asuntos Sociales. Seguridad Social, $N^{\circ}$ 54, p. 53; Holzmann, Robert; Oreinstein, Mitchel y Rutkowki, Michal (eds.) (2003) Pension reform in Europe: Process and Progress, Directions in Development, The Wold Bank, Washington, pp. 47-78; OIT (2003) The gender dimensions of social security reform in central and Eastern Europe: Case study of Czech Republic, Hungary and Poland, Subregional Office for Central and Eastern Europe, Budapest, ILO; MülLER, Katharina (2003); OIT (2002) La reforme des pensions en Europe centrale et orientale. Vol. 1. Restructuration avec privatisation: les cas de la Hongrie et de la Polonie, BIT, Equipe d'Europe centrale et orientale, Budapest. 
canismo. La reforma de 2008 introdujo soluciones a estas brechas de cobertura a través de grandes reformas en el campo de los trabajadores independientes y también en la protección de la mujer. No obstante la importancia de estas reformas, vamos a estudiar el actual modelo chileno desde un punto de vista global. Nuestro análisis considerará cómo las reformas actuales le abren camino al estudio del sistema de pensiones que pasa de un sistema de pilares múltiples a un modelo de una única lógica basado en la capacidad contributiva. Este nuevo avance representa un elemento clave en la búsqueda de dar cobertura a toda la población de esos países que han adoptado el modelo chileno.

Desarrollaremos nuestro análisis en cuatro secciones. En la primera sección presentaremos el cambio de enfoque que se produce a partir de la reforma de 2008, a un nuevo marco de análisis que deberá ser considerado para estudiar el caso chileno. La segunda y tercera sección serán consagradas al análisis de dos elementos del modelo actual: el universalismo y el individualismo. Advertimos desde ya que lo que nos interesa es un enfoque de la lógica del sistema de pensiones resultante de la reforma del 2008, más que un análisis detallado de esta reforma. Y finalmente, en la última sección, presentaremos algunas reflexiones finales.

\section{2) DESDE UN SISTEMA MULTIPILAR A UNA SOLA LÓGICA DE COBERTU- RA}

Actualmente las estrategias para cubrir a la población tienen diferentes características pero su objetivo es el mismo: el riesgo de la vejez. A menudo están formulados por modelos representados por la idea de pilares. El uso de la idea de "pilar de protección" se ha vuelto recurrente en seguridad social. Sin embargo, la imagen de un pilar evoca las columnas que soporta una construcción. Por otro lado, el actual ámbito de aplicación de la seguridad social provee una visión mucho más compleja. Así entonces, a menudo hablamos de múltiples pilares como sistemas de protección para referirnos a una serie de diferentes mecanismos de protección: cobertura diferenciada, pagos según rentas percibidas anualmente y/o capitalizaciones, etc.

De una manera general se puede señalar que el modelo de múltiples pilares de seguridad social se presenta como un edificio de múltiples plantas (cobertura vertical), que entrega una cobertura distinta a diferentes grupos de personas (cobertura horizontal). 


\section{GRÁFICO 1}

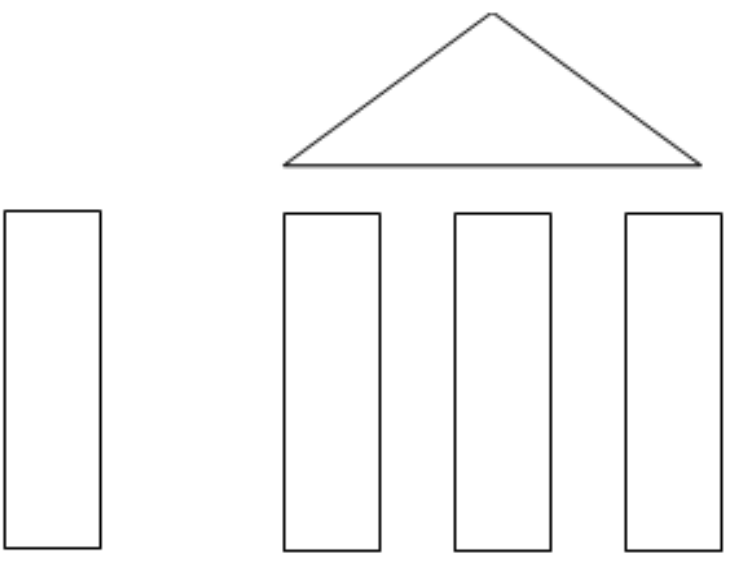

La imagen de un pilar como estructura vertical no parece ser el que debería ser usado en seguridad social cuando se refiere a los sistemas de pensiones. Sin embargo, otras áreas han usado para representar las fundaciones de sus sistemas. Este es el caso de la Unión Europea con la estructura de tres pilares con un techo como representación de los pilares de la construcción del derecho europeo (ver gráfico 1).

Para poder evaluar la cobertura del sistema de pensiones en el contexto de la crisis de envejecimiento de la población; el Banco Mundial ${ }^{5}$ y la Organización Mundial del Trabajo ${ }^{6}$ han propuesto dos modelos de co-

\footnotetext{
5 Acerca este tema ver: World Bank (2005) Old age income support in the 21st Century: An International Perspective on Pension Systems and Reform. World Bank; y también World Bank (1994) Averting the Old Age Crisis: Policies to Protect the Old and Promote Growth. World Bank, Oxford University Press; Roberts, Lucy (1997) "La retraite entre épargne individuelle et assistance: la recette de la Banque mondiale". Chronique Internationale de l'IRES n 48, Septembre 1997, numéro spécial, L'avenir des retraites, p. 17; James, Estelle (1997) "New systems for old age security. Theory, practice, and empirical evidence". Policy research working paper $\mathrm{N}^{\circ} 1766$, Poverty and human resources division, The World Bank, May 1997; James, Estelle (1997) "The World Bank's three-Pillar System: will it provide income security to the World's aging population?”. Economic Studies Working Paper Series $\mathrm{N}^{\circ}$ 26, American Institute for Contemporary German Studes, The Johns Hopkins University, March 1997; James, Estelle (1996) "Fournir une meilleure protection et faciliter la croissance: argument en faveur du rapport La crise du vieillissement". Revue internationale de sécurité sociale, vol. 49, N³, p. 3; JAMEs, Estelle (1995) "Réforme des retraites. Comment parer à la crise du vieillessement”. Finances \& Développement, juin 1995, volume 32, numéro 2, p. 4.

6 ILO (2000) Social security pensions. Development and reform (International Labour Office, Geneva, p. 27.
} 
bertura llamados de "multipilares". El sistema chileno, luego de la reforma de 2008, sin embargo, a nuestro parecer se aleja del modelo de varios pilares, de modo que ahora tiene un solo pilar con dos componentes: un componente contributivo, en el que encontramos las cuentas individuales de ahorro; y el segundo componente, financiado de forma no contributiva por impuestos, constituido por la reciente incorporación de un "sistema de pensiones solidarias"7. El componente principal esta basado en un sistema contributivo obligatorio, el cual ha aumentado la disparidad en los beneficios de las pensiones entre los ciudadanos. Estas diferencias, junto con el aumento de precariedad del trabajo como resultado de la globalización y el aumento del sector informal en la creación de fuentes laborales, han hecho esencial el establecimiento de un beneficio universal para proteger la vejez ${ }^{8}$.

El sistema de multipilares puede ser, de hecho, la adición de diferentes niveles de cobertura que pueden ser presentados como muchos pilares en una posición horizontal. Este esquema tendrá tantos niveles como el modelo considere pilares. Por ejemplo puede tener un pilar básico como pagos según rentas percibidas anualmente, otro de contribuciones definidas y otro de beneficios definidos. En este sentido los pilares ya no estarían representados en forma vertical, sino que horizontal, entregando cada uno una sumas de capas o niveles de cobertura (ver gráfico 2).

\section{GRÁFICO 2}

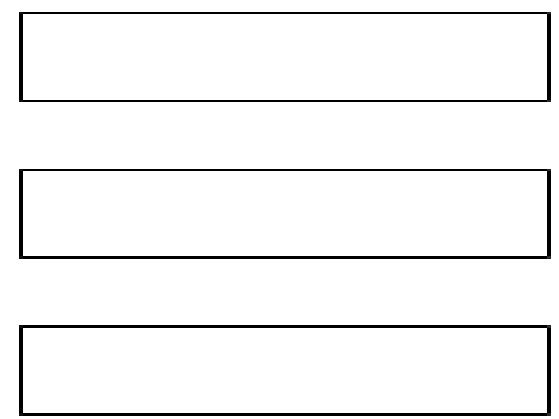

Sin embargo, ninguno de estos pilares entrega una cobertura universal, además cada pilar constituye una cobertura de una sola lógica y cada pilar tiene un campo de aplicación de su cobertura limitado por los

\footnotetext{
$7 \quad$ Antes de la reforma de 2008, el régimen también consideraba dos partes, una con cotización y otra sin cotización, ver: Cifuentes Lillo, Hugo (2005) "Características generales del Sistema de Seguridad Social Chileno, I Parte”. Revista Laboral Chilena, Junio 2005, p. 62.

8 En esto ver: CEPALC (2006); Uthoff (2006) 9; Tielman y Uthoff (2003) 103.
} 
salarios de los trabajadores que pueden participar. En general, se puede señalar que un mecanismo de pensiones multipilares no satisface la universalidad subjetiva.

Esta reforma sugiere, erróneamente, que se trata de la introducción de un pilar de solidaridad. Sin embargo, la solidaridad aquí no es idéntica a la que existe en los sistemas públicos de los países de Europa. Por otro lado, tanto el Banco Mundial como la OIT tienen propuestos modelos de múltiples pilares con un "pilar básico" o "pilar cero" como "fundación" para construir un sistema de protección para la vejez, concebido como una vía para proteger a la parte más vulnerable de la población. En un sistema de pilares, cada pilar responde a una lógica diferente y cubre a una población diferente. La idea de proteger a una población en situaciones precarias es, sin duda, el origen del sistema de pensiones solidarias en Chile. Pero en nuestra opinión el sistema chileno de pensiones tiene un solo pilar basado en la capacidad de ahorro individual, porque los beneficios llamados solidarios solo intervienen cuando la incapacidad de ahorro individual es parcial o inexistente. Así, de esa manera, se ha establecido en el sistema chileno que el paso de un mecanismo a otro depende de la capacidad de ahorro (grande, pequeña o inexistente) de un individuo en un determinado momento. La lógica del sistema chileno es la capacidad de ahorro individual que determina la intervención del Estado en forma total, parcial o no interviene del todo. Así, si representamos esto en un gráfico, en el centro del círculo debemos poner a la lógica del sistema (capacidad de ahorro) que reenvía hacia una parte u otra del sistema. Ya sea hacia el componente no contributivo o ya sea hacia el componente contributivo en sus dos variantes: obligatorio y voluntario (gráfico 3).

\section{GRÁFICO 3}

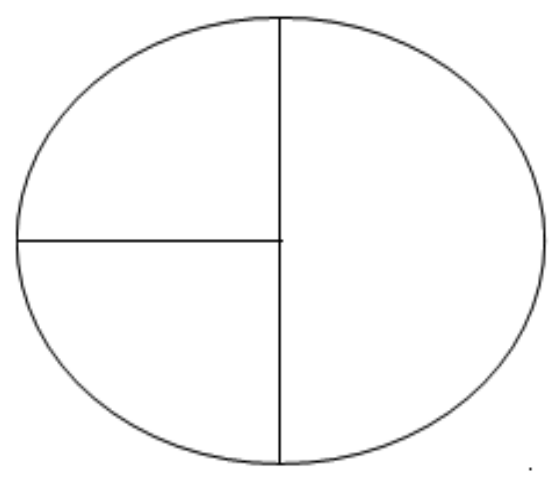


Para construir nuestro marco de análisis, hay algunos principios legales fundamentales, principios que en el caso de Chile son estructurales y operan en forma dinámica estimulando el trabajo legislativo del país. Estos principios son esenciales para la seguridad social de Chile?. La doctrina y leyes chilenas reconocen estos principios, pero no hay acuerdos sobre la manera en que deben interactuar. Sin embargo, consideraremos como principios fundamentales en Chile a los principios, de la seguridad social de: universalidad, solidaridad, comprensión, eficiencia, consistencia y subvención ${ }^{10}$. Para los propósitos de este trabajo solo desarrollaremos en profundidad el principio de universalidad. Este principio puede ser considerado objetivamente, según la cual todas las contingencias sociales están cubiertas. Pero también hay una concepción subjetiva de universalidad, según esta, todos los ciudadanos o residentes deben ser protegidos. Es decir, toda la población está cubierta, pero esa cobertura se logra por medios de beneficios mínimos. Chile ha optado por la universalidad subjetiva: en principio, toda la población está cubierta, pero la cobertura está sujeta a condiciones para obtener beneficios básicos ${ }^{11}$. El principio de universalidad subjetiva en nuestro ordenamiento es el resultado de una combinación de mecanismos públicos y privados, todos basados en la capacidad de ahorro. El análisis de los derechos de pensión incluye necesariamente el análisis de todo el sistema de pensiones y no solo el mecanismo de financiamiento de pensiones.

Tradicionalmente el financiamiento del sistema de pensiones en Chile ha sido clasificado como un sistema que encaja en la estructura de múltiples pilares elaborada por el Banco Mundial. Hoy en día, con la reforma de 2008, esta afirmación se ve disminuida. Por un lado los elementos del modelo de pensiones de Chile puede encajar fácilmente en un esquema de múltiples pilares donde las pensiones solidarias es el "pilar cero" (que puede beneficiar al $60 \%$ de la población), la capitalización obligatoria seria el pilar obligatorio, las contribuciones voluntarias, tanto individuales como colectivas constituyen un segundo pilar, pero secundario y complementario al pilar obligatorio. Pero desde otro punto de vista, es posible discutir que el sistema chileno ya no sigue la estructura del modelo de múltiples pilares y constituye un sistema único de protección contra la

9 Lanata, Gabriela (1999) Manual de legislación previsional. Proyecto de Desarrollo de la Docencia, Universidad de Concepción, p. 9.

10 Acerca de los principios de seguridad social ver: Novoa Fuenzalida, Patricio (1977) Derecho de la Seguridad Social. Editorial Jurídica de Chile, Santiago; Hormazábal, Ricardo (2007) "El sistema de AFP chileno: una visión critica". Revista Chilena de Administración Pública, $\mathrm{n}^{\circ} 9$ Junio 2007, p 123; Lanata (1999) 9; Gumucio, Juan Sebastián (1996) "De reparto a capitalización: la experiencia chilena, II Parte". Revista Laboral Chilena, Noviembre, pp. 66-74; Valdés-Prieto, Salvador (2002) Políticas y mercados de pensiones. Un texto Universitario para América latina. Ediciones Universidad Católica de Chile, Santiago, p. 36. 
vejez $^{12}$. Mientras hay diferentes mecanismos (no contributivo, contribución obligatoria y voluntaria), el sistema de pensiones chileno tiene una lógica global: la capacidad individual de ahorro. Esta capacidad determina qué mecanismo se aplicará para cada caso particular, así, una persona sin capacidad para ahorrar recibirá una pensión básica solidaria; una persona con poca o moderada capacidad ahora recibirá un aporte solidario; una persona que tiene una gran capacidad de ahorro hará contribuciones en su cuenta de capitalización individual obteniendo una pensión de su respectiva AFP; y por último, quienes poseen una capacidad contributiva más allá de lo establecido como obligatorio podrán hacer cotizaciones voluntarias y mejorar sus pensiones.

\section{3) UNIVERSALISMO}

El derecho a la seguridad social está consagrado en la Constitución de 1980 con bastante certeza, aunque todavía lejos de una completa consagración como otros textos constitucionales ${ }^{13}$. El texto chileno establece que el Estado debe fijar un objetivo para garantizar el acceso a todos los residentes y disfrutar los beneficios básicos uniformes. Los beneficios serán reconocidos por instituciones públicas o privadas ${ }^{14}$. La Constitución agrega que el Estado debería monitorear el apropiado ejercicio de este derecho. El artículo $19 \mathrm{~N}^{\circ} 18$ de la Constitución consagra el derecho a la seguridad social: "El derecho a la seguridad social. Las leyes que regulen el ejercicio de este derecho serán de quórum calificado.

La acción del Estado estará dirigida a garantizar el acceso de todos los habitantes al goce de prestaciones básicas uniformes, sea que se otorguen a través de instituciones públicas o privadas. La ley podrá establecer cotizaciones obligatorias. social".

El Estado supervigilará el adecuado ejercicio del derecho a la seguridad

Por otro lado, la Constitución de $1980^{15}$ en su artículo $5^{\circ}$ párrafo 2 establece un mecanismo para el reconocimiento de derechos humanos internacionales como normas aplicables en derecho interno que nos obli-

12 Más detalles sobre esta opinión: Arellano Ortiz, Pablo (2009) Universalisme et individualisme dans le droit chilien des retraites, Thèse, Université Paris Ouest La Défense; Arellano Ortiz, Pablo (2008) "El pilar solidario, la solución esperada para nuestro sistema de AFP". Revista Laboral Chilena, Febrero- Marzo, $\mathrm{N}^{\circ} 164$, pp. 86-88.

13 Por ejemplo, la Constitución del Estado Plurinacional de Bolivia del año 2009.

14 Cifuentes Lillo (2005) 61; ver también: Ribera Neumann, Teodoro (2002) "El derecho a la seguridad social en la jurisprudencia del Tribunal Constitucional: Comentarios a la sentencia ROL N ${ }^{\circ} 334$ ". Revista de Derecho, Universidad de Concepción, N²12, Año LXXI, Julio-Septiembre, pp. 179-201.

15 D.O. 24 de octubre de 1980, con reforma de la ley 18.825 D.O. de 17 de Julio de 1989. 
ga a considerar la cuestión de fortalecer estas normas dentro del derecho nacional. Por lo tanto, gracias a esta norma se puede afirmar que en Chile opera un bloque de constitucionalidad que actúa como un bloque dinámico que nos acerca hacia los estándares internacionales. De esta manera se puede llegar a establecer la recepción efectiva de las obligaciones internacionales sobre universalidad de la cobertura en el derecho nacional. El texto original de este artículo de la Constitución ha sido modificado y hoy se consagra de la siguiente forma: "La soberanía reside esencialmente en la Nación. Su ejercicio se realiza por el pueblo a través del plebiscito y de elecciones periódicas y, también, por las autoridades que esta Constitución establece. Ningún sector del pueblo ni individuo alguno puede atribuirse su ejercicio.

El ejercicio de la soberanía reconoce como limitación el respeto a los derechos esenciales que emanan de la naturaleza humana. Es deber de los órganos del Estado respetar y promover tales derechos, garantizados por esta Constitución, asi como por los tratados internacionales ratificados por Chile y que se encuentren vigentes".

Es gracias, entonces, a este artículo de la Constitución que las fuentes del derecho a la seguridad social, tanto internacionales como constitucionales, pueden ser entendidas en una relación de interacción que permite exigir una recepción y exigibilidad amplia del derecho a la seguridad social en nuestro ordenamiento. Siguiendo esta línea de análisis tenemos por ejemplo el texto de la Declaración Universal de Derechos Humanos de las Naciones Unidas y el de la Organización de los Estado Americanos, que han sido importantemente estudiados por la doctrina chilena. Hay un concepto de derechos humanos en la legislación chilena gracias al rol que ha tenido el bloque constitucional, el cual incluye a la seguridad social como un derecho humano.

Sin embargo, estas obligaciones constitucionales e internacionales deben tener un mecanismo de fortalecimiento en el derecho nacional. Así, de esta manera, mecanismos de fortalecimiento de los derechos sociales de la ley chilena muestran que la seguridad social chilena ha evolucionado a un sistema en donde los conceptos de riesgo social ahora no solo dependen de las instituciones del gobierno, sino también de organizaciones privadas. Esto ha sido el resultado de un reciente desarrollo de la jurisprudencia del Tribunal Constitucional de Chile ${ }^{16}$ que ha aplicado

16 Caso "Isapres" ROL n 976-07, de 26 de junio de 2008. 
la teoría alemana ${ }^{17}$ de exigibilidad ${ }^{18}$ horizontal de derechos fundamentales ${ }^{19}$.

El requerimiento de realización en el Derecho nacional de protección por el Estado se ha convertido en una realidad a través de la reforma de 2008.

Así, de este modo, el sistema de pensión solidaria consolidado, armonizó y mejoró el régimen asistencial de pensiones y el régimen de pensiones mínimas garantizadas, las cuales existieron antes del establecimiento de la ley 20.255. Estos mecanismos sufrieron desde el comienzo de críticas, se trataba de programas fuertemente dependientes de los presupuestos del gobierno, de bajos recursos y con un largo déficit de cobertura y que no fueron designados a dar derechos, sino solo beneficios condicionales. El nuevo mecanismo no contributivo provee dos tipos de beneficios basados en un derecho legal. Los beneficios introducidos y que realizan el derecho a la seguridad social, y por ende el universalismo, se pueden resumir de la siguiente manera:

\section{1.) LA PENSIÓN SOLIDARIA BÁSICA}

La pensión solidaria ${ }^{20}$ es financiada por el presupuesto general del Estado, es decir, un mecanismo de carácter no contributivo, y administrada por el Instituto de Previsión Social (IPS). Este nuevo mecanismo que se agrega al sistema de pensiones chileno ${ }^{21}$ constituye una base sólida de la protección de vejez. Esta reforma consagra una pensión mínima para

17 Esta teoría del efecto horizontal con origen en la doctrina alemana del Drittwirkung, cuyo efecto ha sido deducido del famoso caso "Lüth" de la Corte Constitucional Alemana, BVerfGE 7, 198 (205), decisión del 15 de enero de 1958. Sobre este efecto ver: Favoreu, Louis; Gaïa, Patrick; Ghevontian, Richard; Mestre, Jean-Louis; Pfersmann, Otto; Roux, André y Scoffoni, Guy (2008) Droit constitutionnel, 1iéme édition, Précis, Dalloz, Paris, pp. 861 y 881; JouANJan, Olivier (1998) «La théorie allemande des droits fondamentaux». AJDA, p. 44.

18 Nosotros preferimos la utilización del término exigibilidad a diferencia de una parte de la doctrina chilena que se refiere a la teoría alemana como de la eficacia horizontal. Para nosotros, el término eficacia no es el más apropiado, ya que es de común uso en análisis económico del derecho para contraponerlo con el término efectividad.

19 Martínez Estay, José Ignacio (1998) "Los particulares como sujetos pasivos de los derechos fundamentales: La doctrina del efecto horizontal de los derechos". Revista Chilena de Derecho, 1998, Número especial, p. 62.

20 La reforma incluye también una pensión solidaria de invalidez y de sobrevivencia, de las cuales no trataremos.

21 Mesa-Lago, Carmelo (2008) "La protection sociale au Chili: des réformes pour plus de justice". Revue internationale du travail, vol. 147, $\mathrm{n}^{\circ}$ 4, p. 428; WALKer y Cifuentes (2008); ver de los mismos autores: (2007) "Principales aspectos institucionales del Proyecto de Ley que perfecciona el Sistema de Pensiones". Revista de Economía y Administración, Facultad de Economía y Negocios, Universidad de Chile, Mayo, N 154, pp. 58-69. 
una amplia mayoría de los chilenos, las prestaciones están destinadas a aquellos quienes cumplan con ciertas condiciones: las personas de edad, nivel socioeconómico bajo y residencia durante un período determinado. Todos aquellos que cumplen las condiciones, se benefician de una pensión básica solidaria financiada por el Estado, lo que en doctrina es conocido como una pensión no contributiva. Para tener derecho al sistema básico de pensión solidaria, las personas deben encontrarse en las siguientes condiciones:

i) No tener derecho a una pensión bajo ningún régimen.

ii) Que la persona tenga al menos 65 ańos de edad, la edad de jubilación legal para hombres hoy en día y para las mujeres es de 60 años de edad. Entonces una mujer para recibir los beneficios debe esperar 5 años más que si ella se retirara en un mecanismo de fondos de cotización. Sin embargo, creemos que esta medida tiene por objeto evitar la discriminación de edad y aumentar en un futuro próximo la edad de jubilación de las mujeres a los 65 ańos.

iii) Que la persona sea parte de un grupo familiar ${ }^{22}$ perteneciente al $60 \%$ más pobre de la población, esta prestación entonces no es universal, ella protege a una parte importante, pero no a toda la población.

iv) Justificar residencia en el territorio nacional chileno de por al menos 20 ańos, ya sea continua o discontinua, de los cuales cuatro deben estar incluidos en los últimos cinco ańos inmediatamente anteriores a la solicitud de la pensión solidaria. Este criterio de residencia nos permite decir que la prestación actualmente está vinculada a la ciudadanía chilena, y más amplio aún, porque un extranjero con una residencia de 20 años también tiene derecho. Sin duda esto representa un importante progreso en temas de extensión de protección social. Este periodo de 20 años de residencia, se cuenta a partir del vigésimo aniversario de la persona, excepto aquellos casos calificados como sin recursos, para los que este período se cuenta desde la fecha de nacimiento ${ }^{23}$.

El monto de la pensión básica solidaria a partir del 1 de julio de 2009 será de $\$ 75.000^{24}$, y será incompatible con otra pensión de otro régimen previsional. La pensión se reajustará automáticamente en el 100\% de la variación del Índice de Precios al Consumidor (IPC), determinado

22 Sobre la noción de familia en seguridad social ver: Arellano Ortiz, Pablo (2012) "La noción de familia en seguridad social: aporte a la discusión del proyecto de ley sobre el Acuerdo de Vida en Pareja". Revista Laboral Chilena, abril 2012, pp. 54-62.

23 Para el caso de los antiguos exiliados que se benefician de la ley $\mathrm{n}^{\circ} 18.994$, se ha establecido que el tiempo pasado en el extranjero era, a pesar de todo, contabilizado, Art. 5 de la ley $n^{\circ}$ 20.255 .

24 Existe un periodo transitorio entre el 1 de julio de 2008 hasta el 30 de junio de 2009 durante el cual el monto de esta pensión es de $\$ 60.000$, ver artículo decimotercero del párrafo primero, del Título VIII «Sobre las disposiciones transitorias» de la ley nº 20.255 . 
por el Instituto Nacional de Estadísticas; el primer reajuste se aplicó a partir del 1 de julio de 2009.

\section{2.) EL APORTE SOLIDARIO}

Es la segunda prestación incorporada al sistema por la reforma bajo la forma de un aporte mensual ${ }^{25}$. Este aporte tiene como objetivo completar las cantidades de las pensiones concedidas en virtud del DL $\mathrm{N}^{\circ}$ 3.500 de 1980, cuando están por debajo de un nivel de renta determinada de acuerdo con las normas del mismo decreto.

Para acceder a esta prestación, la ley ha establecido las siguientes condiciones:

i) Tener derecho a una o más pensiones contenidas en el DL 3.500 de 1980.

ii) Que el monto de la pensión sea menor al valor máximo de una pensión con aporte solidario. De hecho, se trata de un aporte a monto variable.

iii) Se debe cumplir con todas las condiciones exigidas para acceder a la pensión básica solidaria, es decir, tener al menos 65 años, ser parte de un grupo familiar perteneciente al $60 \%$ más pobre del país y justificar un período de residencia. El requisito de residencia se cumple cuando el solicitante del aporte es capaz de justificar 20 años de cotizaciones.

El aporte solidario alcanzará la cantidad que resulte después de restar a la pensión final el monto de la o las pensiones que percibe el solicitante del aporte. El aporte solidario disminuye del monto superior de pensión hasta extinguirse al llegar a una pensión de monto igual o superior a $\$ 255.000^{26}$. El aporte será reajustado según el IPC y en la misma forma que para la pensión básica solidaria

Este aporte ha sido concebido con una doble finalidad. En primer lugar, aliviar a la clase media que no puede permitirse ahorrar más allá de la obligación legal, y que fue, por lo mismo, con frecuencia excluida de la

25 Mesa-Lago (2008); Walker y Cifuentes (2008); ver de los mismos autores (2007).

26 Este monto corresponde a la prestación después del período de transición, es decir, a contar del 1 de julio de 2011, la pensión con aporte solidario alcanzará un máximo de \$255.000. El período de transición consiste en varias etapas, a saber, entre el 1 de julio de 2008 y hasta el 30 de junio de 2009 la pensión con el aporte solidario alcanzará un máximo de \$70.000; entre el 1 de julio de 2009 y hasta el 31 de agosto 2009 la pensión con la aporte solidario alcanzará un máximo de \$120.000; del 1 de septiembre de 2009 y hasta el 30 de junio de 2010 la pensión con la aporte solidario alcanzará un máximo de $\$ 150.000$, y entre el 1 de julio de 2010 y hasta el 30 de junio de 2011 la pensión con la aporte solidario alcanzará un máximo de \$200.000. Según lo establece el artículo XIII, Párrafo primero, del Título VIII, "Disposiciones transitorias" de la Ley No 20.255, modificada por la Ley No 20.366, D.O. 27.07.2009. 
protección. En segundo lugar, permitir a todos los excluidos, finalmente, contar con una pensión, incluso si sus ahorros son bajos.

También se puede apreciar que con este tipo de mecanismos se conjugan actores tanto públicos como privados hacia un solo beneficio que administrar, el cual tiene una parte que proviene del Estado (el aporte) y otra parte de los fondos acumulados en las AFP.

Estas prestaciones muestran que el universalismo ha sido reforzado en el sistema de seguridad social chileno. La reforma de 2008 cambió el sistema de pensiones chileno a un sistema híbrido que pretende ofrecer cobertura universal a toda la población. Es un híbrido en lo que a administración, financiamiento y beneficios concierne. Esto se parece al sistema de pensiones sueco, que es demostrado en el gráfico 4. La diferencia con el sistema chileno, es que no hay un mecanismo de cuentas nociona$\operatorname{les}^{27}$.

\section{GRÁFICO 4}

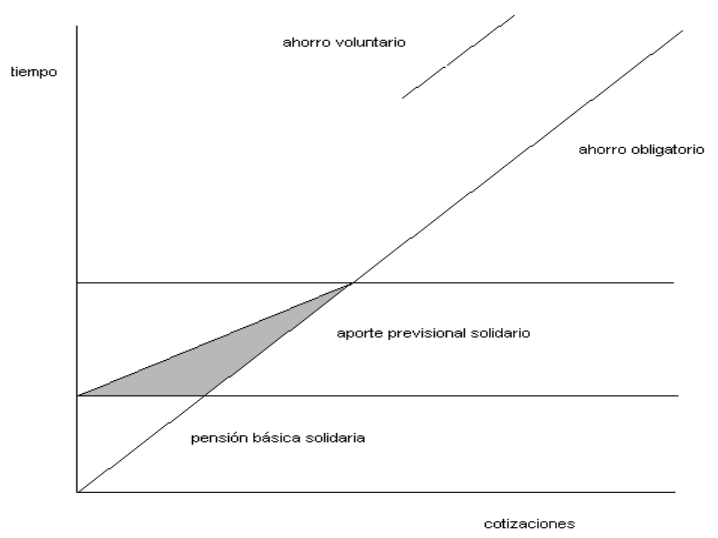

Fuente: Arellano Ortiz, Pablo (2009)

27 Este tipo de mecanismo implica un esquema muy similar el chileno y ha sido llevado acabo en Suecia, más información sobre este tipo de mecanismo ver: COR (2009) Le système de retraite suédois. Mécanismes, enjeux et perspectives. Colloque organisée par le Conseil d'orientation des retraites, le 16 octobre 2008, La documentation Française ; ANxo, D. y NikLasson, H. (2008) «The Swedish model: Revival after the trubulent 1990s?», Document de Travail DP/189/2008, Institut International d'Études Sociales, OIT; Toutain, S. (2007) Le nouvel âge des retraites, Ellipse, CEPALC, 2006, p. 146; PALMer, E. (2006) «El Nuevo sistema de pensiones sueco de cuentas individuales de capitalización", Documento de Trabajo $\mathrm{n}^{\circ}$ 15, Superintendencia de Administradoras de Fondos de Pensiones, Septiembre 2006; Palmer, E. (2001) «L'évolution de l'assurance publique et privée en Suède pendant les années quatre-vingt-dix", in X. SCHeIL-Adlung, Construire la sécurité sociale: la privatisation en question, AISS, Peter Lang SA, Bern, 2001, p. 235. 


\section{4) INDIVIDUALISMO}

Adicionalmente, la reforma de 2008 introdujo una figura que previamente no existía en la legislación de seguridad social chilena, la cual consiste en los planes de ahorro colectivo. Este mecanismo se asemeja a los planes de pensión $401(\mathrm{k})$ de la legislación norteamericana y contiene la posibilidad que el empleador haga una cotización equivalente o mayor a la efectuada por el trabajador (match-contribution) ${ }^{28}$. Este mecanismo de ahorro requiere un importante diálogo y participación entre trabajadores y empleadores en orden a obtener suficientes beneficios. Además, para producir un efecto significante en la mano de obra, es que debe existir un alto nivel de negociación colectiva, situación que no ocurre en Chile $^{29}$. Notamos que la introducción de este mecanismo es un progreso en relación con cotizaciones de los empleadores, las cuales son muy bajas en el sistema chileno de seguridad social. Por otra parte, el Decreto Ley $\mathrm{N}^{\circ} 3.500$ incluye mecanismo de ahorro previsional voluntario individual tales como la figura de Depósitos Convenidos, bajo la cual los trabajadores afiliados pueden depositar dentro de sus cuentas individuales, montos que han sido agregados provisionalmente con el empleador en orden a alcanzar los fondos suficientes para financiar la anticipada jubilación por retiro o incrementar los montos de pensión.

Así, de este modo, hay una creciente participación de los empleadores para financiar el mecanismo contributivo, hoy el empleador está legalmente excluido de contribuir al financiamiento obligatorio de cuentas individuales. Sin embargo, a través de los planes de ahorro colectivo, la participación del empleador en el financiamiento de la pensión es posible y este aporte puede afectar significativamente los montos finales de las pensiones. No obstante, la conformidad con los estándares internacionales aún no es posible ya que instrumentos tales como la Convención $\mathrm{n}^{\mathrm{o}} 102$ de la OIT prescribe que en casos como el chileno el aporte al financiamiento de las prestaciones debería ser compartido entre empleadores y trabajadores. Esto es solo posible, hoy en día, a nivel complementario en Chile y no en el régimen básico obligatorio.

La reforma, además, ha fortalecido de manera importante el componente individual con las correcciones para los trabajadores independientes y para las mujeres. Sin lugar a dudas la reforma, además de extender la cobertura, lo que hace es confirmar al sistema de capitalización individual como el mecanismo básico obligatorio para los trabajadores independien-

Existe la posibilidad que el trabajador puede que no contribuya del todo si ello es fijado así en el pan de pensiones.

29 Gamonal, Sergio (2008) Fundamentos de derecho laboral, LegalPublishing, Santiago, p. 23. 
tes. La reforma no cuestiona dicho mecanismo, sino que lo corrige y lo valida.

\section{5) CONCLUSIONES}

La seguridad social en materia de pensiones está cambiando hoy en día y sobre todo en Chile. De las correcciones aportadas por la reforma de 2008 al sistema de pensiones chileno, un número importante de conclusiones se pueden extraer. A nuestro parecer la idea de poder concebir la protección de la vejez desde un punto de vista global a través del marco de análisis propuesto, basado en una sola lógica, la capacidad contributiva, permite al modelo chileno de pensiones poder ajustarse con mayor claridad a los principios básicos de la seguridad social. Pero no solo nuestro país se ve beneficiado con la adopción de un modelo basado en una sola lógica. Si consideramos a los países de Europa del Este, la incorporación de este tipo de modelo podría permitir a éstos facilitar su integración en los procesos de coordinación de mecanismos de seguridad social europeos y, por ende, su integración más completa dentro de la Unión Europea $^{30}$.

Una cuestión importante en relación con los mecanismos de pensión como el modelo chileno es la conformidad con los estándares internacionales, en particular con los principios de seguridad social establecidos por el Convenio $\mathrm{N}^{\circ} 102$ de OIT. A este respeto, nuestro país ha sido objeto en numerosas ocasiones de observaciones por parte de la Comisión de Expertos en la Aplicación de Convenios y Recomendaciones de la OIT. Más aún, el Consejo Administrativo ${ }^{31}$ de la OIT, en marzo de 2000, ha pronunciado una serie de recomendaciones para que nuestro país se logre alinear con los estándares internacionales ${ }^{32}$, a saber: (i) que el sistema de

30 Uno de los problemas de la coordinación europea en materia de seguridad social es la necesidad de encontrar un mecanismo para coordinar los diferentes sistemas de cobertura de la protección de la vejez ya sea fondos de pensión o los regímenes de reparto. Hoy, esta coordinación no es posible desde que no hay mecanismos que puedan establecer una pensión para un trabajador que ha trabajado en países con régimen de reparto y también en países que poseen fondos de pensiones como regímenes básicos. El problema es el contraste de legislación entre Europa del Oeste y del Este. En el lado oeste de Europa la mayoría de los países tienen un sistema de reparto, y en el otro lado, la Europa del Este, hay un importante numero de países que siguieron el modelo chileno y tienen fondos de pensiones privados como régimen básico de seguridad social. Ver: Arellano Ortiz, Pablo (2012a) "Universalism and Individualism in Chilean pension law: an example of extension of coverage for Eastern Europe”. En Roger Blanpain, William Bromwich, Olga Rymkevich \& Iacopo Senatori (eds.), Bulletin of Comparative Labour Relations Vol. 80 "Labour Markets, Industrial Relations and Human Resources Management". Kluwer. pp. 219-231. ISI.

31 OIT (2010) Governing Body, 277th Session, March 2000 (GB.277/17/5, March 2000).

32 Estas recomendaciones fueron pronunciadas en relación con el Convenio $\mathrm{N}^{\circ} 35$ ya que nuestro país aún no ratifica el Convenio $\mathrm{N}^{\circ} 102$. 
pensión, establecido en 1980 por el Decreto Ley No 3.500, debería ser administrado por organizaciones sin fines de lucro; (ii) que representantes del asegurado deberían participar en la administración del sistema bajo condiciones determinadas por el derecho nacional y la práctica; (iii) los empleadores deberían contribuir al financiamiento del sistema de seguro. Estas recomendaciones son aun válidas para Chile después de la reforma de 2008; el mecanismo de fondos de pensiones no ha sido reformado ${ }^{33}$.

A nuestro parecer, estas observaciones de la OIT, podrían ser evitadas, corregidas y superadas si se optara por un marco de análisis basado en un modelo de una sola lógica, como el que sostenemos que tiene Chile de hoy en día. En esta forma de comprender un sistema de pensiones: la administración pasa a ser pública, los asegurados participan a través de la Comisión de Usuarios, y hay avances en los aportes de los empleadores. Más aún, gracias a este nuevo enfoque el Gobierno chileno podría ratificar el Convenio $\mathrm{N}^{\circ} 102$ sobre norma mínima, ya que a nuestro entender se cumple con dicho estándar internacional.

El modelo individual establecido en Chile en el año 1980 ha mostrado muchos problemas de cobertura y de conformidad con los estándares internacionales por muchos ańos. Sin embargo, la reciente reforma del año 2008 posibilita corregir las brechas de cobertura y otorga la posibilidad de estar en línea con los estándares de la OIT, especialmente con el Convenio $\mathrm{N}^{\circ} 102$. Se puede señalar que la privatización parece estar en retirada en América Latina ${ }^{34}$, tendencia que puede ser seguida por todos los países europeos que tienen un sistema de capitalización privada. Finalmente, la adopción de este único marco podría ayudar a dar coherencia al sistema de seguridad social chileno. Esta coherencia podría ser la clave para una mayor y mejor cobertura.

\section{BIBLIOGRAFÍA}

- Anxo, D. y Niklasson, H. (2008) «The Swedish model: Revival after the trubulent 1990s?», Document de Travail DP/189/2008, Institut International d'Études Sociales, OIT.

- Arellano Ortiz, Pablo (2012a) "Universalism and Individualism in Chilean pension law: an example of extension of coverage for

\footnotetext{
33 Hemos desarrollado estos argumentos en: Arellano Ortiz, Pablo y Cifuentes Lillo, Hugo (2010) «Legislación chilena de pensión e indicaciones de la Comisión de Expertos de la OIT» Revista Chilena de Derecho de Trabajo y de la Seguridad Social, Departamento de Derecho de Trabajo y de la Seguridad Social, Facultad de Derecho, Universidad de Chile, Vol. 1, $\mathrm{N}^{\circ} 1$, pp. 123-130.

34 Calvo, Esteban; Bertranou, Fabio y Bertranou, Evelina. (2010) 'Are Old-age Pension System Reforms Moving Away from Individual Retirement Accounts in Latin America?' Journal of Social Policy, Volume 39, Number 2, pp. 223-234.
} 
Eastern Europe”. En Roger Blanpain, William Bromwich, Olga Rymkevich \& Iacopo Senatori (eds.), Bulletin of Comparative Labour Relations Vol. 80 "Labour Markets, Industrial Relations and Human Resources Management”. Kluwer, pp. 219-231.ISI

- Arellano Ortiz, Pablo (2012b) "La noción de familia en seguridad social: aporte a la discusión del proyecto de ley sobre el Acuerdo de Vida en Pareja”. Revista Laboral Chilena, abril 2012, pp. 54-62.

- Arellano Ortiz, Pablo y Cifuentes lillo, Hugo (2010) «Legislación chilena de pensión e indicaciones de la Comisión de Expertos de la OIT» Revista Chilena de Derecho de Trabajo y de la Seguridad Social, Departamento de Derecho de Trabajo y de la Seguridad Social, Facultad de Derecho, Universidad de Chile, Vol. 1, $\mathrm{N}^{\circ} 1$, pp. 123-130.

- Arellano Ortiz, Pablo (2009) Universalisme et individualisme dans le droit chilien des retraites, Thèse, Université Paris Ouest La Défense.

- Arellano Ortiz, Pablo (2008) "El pilar solidario, la solución esperada para nuestro sistema de AFP". Revista Laboral Chilena, Febrero-Marzo, No 164, pp. 86-88.

- Bertranous, Fabio, Rofman, Rafael y Grushka, Carlos (2003) «De la réforme à la crise: le système de pensions en Argentine». Revue internationale de sécurité sociale, vol. 56, $\mathrm{N}^{\circ} 2$, p. 129.

- Bertranous, Fabio y Rofman, Rafael (2002) «La prestation de sécurité sociale dans un contexte en plein évolution: l'expérience et les défis de l'Amérique latine». Revue internationale de sécurité sociale, vol. $55, \mathrm{~N}^{\circ} 1$, p. 75 .

- Bonilla, Alejandro y Conte-Grand, Alfredo (1999) «Reforma de pensiones en América latina: desafíos pendientes, estrategias para el futuro». Análisis laboral, Marzo, p. 10.

- Bonilla, Alejandro y Conte-Grand, Alfredo (eds.) (1998) Pensiones en América Latina. Dos décadas de reforma. Ginebra: OIT.

- Calvo, Esteban; Bertranou, Fabio y Bertranou, Evelina. (2010) 'Are Old-age Pension System Reforms Moving Away from Individual Retirement Accounts in Latin America?', Journal of Social Policy, Volume 39, Number 2, pp. 223-234.

- Cifuentes Lillo, Hugo (2008a) Reforma previsional. Santiago: LexisNexis.

- Cifuentes Lillo, Hugo (2008b) "Comentario sobre la reforma previsional chilena de 2008". Revista de la red de expertos iberoamericanos en seguridad social, Ceddet, OISS, No 2, primer semestre de 2008, pp. 8-12.

- Cifuentes Lillo, Hugo (2005) "Características generales del Sistema de Seguridad Social Chileno, I Parte”. Revista Laboral Chilena, Junio 2005, p. 62. 
- CEPALC (2006) La protección social de cara al futuro: acceso, financiamiento y solidaridad. Santiago : CEPALC, Naciones Unidas.

- COR (2009) Le système de retraite suédois. Mécanismes, enjeux et perspectives. Colloque organisée par le Conseil d'orientation des retraites, le 16 octobre 2008, La documentation Française.

- Favoreu, Louis; Gaïa, Patrick; Ghevontian, Richard; Mestre, Jean-Louis; Pfersmann, Otto; Roux, André y Scoffoni, Guy (2008) Droit constitutionnel, 1e édition, Paris: Précis, Dalloz,

- FIAP (2004) La reforma de pensiones en Europa del Este: Experiencias $y$ Perspectivas. Fiap, CIEDESS.

- Fuenzalida, Patricio (1977) Derecho de la Seguridad Social. Santiago: Editorial Jurídica de Chile.

- Gamonal, Sergio (2008) Fundamentos de derecho laboral, LegalPublishing, Santiago.

- Gill, Indermit; Packard, Truman; Pugatch, Todd y Yermo, Juan (2005) «Repenser la sécurité sociale en Amérique latine». Revue internationale de sécurité sociale, vol. $58, \mathrm{~N}^{\circ} 2 / 3$, p. 81 .

- Gumucio, Juan Sebastián (1996) "De reparto a capitalización: la experiencia chilena, II Parte". Revista Laboral Chilena, Noviembre, p. 66-74.

- Gumucio, Juan Sebastián (1977) «Chile en el Mercosur : armonización de sistemas de seguridad social». Revista Laboral Chilena, Enero 1977, pp. 58-64.

- Holzmann, Robert; Oreinstein, Mitchel y Rutkowki, Michal (eds.) (2003) Pension reform in Europe: Process and Progress, Directions in Development. Washington: The Wold Bank.

- Hormazábal, Ricardo (2007) "El sistema de AFP chileno: una visión critica". Revista Chilena de Administración Pública, $\mathrm{n}^{\circ} 9$ Junio 2007, p 123.

- James, Estelle (1997) "New systems for old age security. Theory, practice, and empirical evidence". Policy research working paper $N^{\circ}$ 1766, Poverty and human resources division, The World Bank, May 1997.

- James, Estelle (1997) "The World Bank's three-Pillar System: will it provide income security to the World's aging population?". Economic Studies Working Paper Series $N^{\circ}$ 26, American Institute for Contemporary German Studes, The John Hopkins University, March 1997.

- James, Estelle (1996) "Fournir une meilleure protection et faciliter la croissance: argument en faveur du rapport La crise du vieillissement". Revue internationale de sécurité sociale, vol. 49, $\mathrm{N}^{\circ} 3$, p. 3 . 
- James, Estelle (1995) "Réforme des retraites. Comment parer à la crise du vieillessement". Finances \& Développement, juin 1995, volume 32, numéro 2 , p. 4.

- Lanata, Gabriela (1999) Manual de legislación previsional. Proyecto de Desarrollo de la Docencia, Universidad de Concepción.

- Lancereau, Thomas (1999) «Les fonds de pension en Amérique latine. Les fonds de pension latino-américains produiront-ils les effets que l'on prête au modèle chilien?» Futuribles, juillet - août, p. 109.

- Martínez Estay, José Ignacio (1998) "Los particulares como sujetos pasivos de los derechos fundamentales: La doctrina del efecto horizontal de los derechos". Revista Chilena de Derecho, 1998, Número especial, p. 62.

- Matijascic, Milko y Kay, Stephen J. (2006) «La sécurité sociale à un carrefour: vers une réforme effective des pensions en Amérique Latine». Revue internationale de sécurité sociale, vol. 59, $\mathrm{N}^{\circ} 1$, p. 3.

- Mesa-Lago, Carmelo (2008) "La protection sociale au Chili: des réformes pour plus de justice". Revue internationale du travail, vol. $147, \mathrm{n}^{\circ} 4$, p. 428.

- Mesa-Lago, Carmelo (2005) «Les réformes de la sécurité sociale en Amerique latine: observations sur le rapport de 2005 de la Banque Mondiale». Revue internationale de sécurité sociale, Vol. 58 No 2-3, p. 115

- Müller, Katharina (2003) «The making of pension privatisation in Latin America and Eastern Europe». En Holzmann, Robert; Oreinstein, Mitchel y Rutkowki, Michal (eds.) (2003) Pension reform in Europe: Process and Progress, Directions in Development, The Wold Bank, Washington, pp. 47-78.

- Ochando Claramunt, Carlos (2004) "Evaluation des réformes des systèmes de pensions latino-américaines", Revue internationale de sécurité sociale, vol. $57, \mathrm{~N}^{\circ} 2$, p. 33.

- OIT (2010) Governing Body, 277th Session, March 2000 (GB.277/17/5, March 2000).

- OIT (2006) Pension reform in the Baltic States. Estonia - Lativia Lithuania, Subregional Office for Central and Eastern Europe, Budapest, ILO.

- OIT (2005) Reforma de las pensiones en Europa central y oriental, Oficina subregional para Europa Central y Oriental, BIT, Budapest.

- OIT (2003) The gender dimensions of social security reform in central and Eastern Europe : Case study of Czech Republic, Hungary and Poland, Subregional Office for Central and Eastern Europe, Budapest, ILO. 
- OIT (2002) La reforme des pensions en Europe centrale et orientale. Vol. 1. Restructuration avec privatisation: les cas de la Hongrie et de la Polonie, BIT, Equipe d'Europe centrale et orientale, Budapest.

- OIT (2000) Social security pensions. Development and reform (International Labour Office), Geneva.

- Palmer, E. (2006) «El Nuevo sistema de pensiones sueco de cuentas individuales de capitalización», Documento de Trabajo $\mathrm{n}^{\circ}$ 15. Superintendencia de Administradoras de Fondos de Pensiones, Septiembre 2006.

- Palmer, E. (2001) «L'évolution de l'assurance publique et privée en Suède pendant les années quatre-vingt-dix», in X. ScheIL-AdLung, Construire la sécurité sociale: la privatisation en question, AISS, Peter Lang SA, Bern, 2001, p. 235.

- Roberts, Lucy (1997) "La retraite entre épargne individuelle et assistance: la recette de la Banque mondiale". Chronique Internationale de l'IRES $n^{\circ} 48$, Septembre 1997, numéro spécial, L'avenir des retraites, p. 17.

- Jounjuan, Olivier (1998) «La théorie allemande des droits fondamentaux». AJDA, p. 44

- Queisser, Monika (1995) "Apres le Chili, les reformes de la deuxième génération en Amérique Latine". Revue internationale de sécurité sociale, vol. 48, 3/4, p. 27.

- Ribera Neumann, Teodoro (2002) «El derecho a la seguridad social en la jurisprudencia del Tribunal Constitucional: Comentarios a la sentencia ROL N ${ }^{\circ} 334 \%$. Revista de Derecho, Universidad de Concepción, N² 212, Año LXXI, Julio-Septiembre, pp. 179-201.

- Tielman, Daniel y Uthoff, Andras (2005) «Assurance et protection sociale en Amérique latine', Revue internationale de sécurité sociale, vol. $58, \mathrm{~N}^{\circ} 2 / 3$, p. 49.

- Tielman, Daniel y Uthoff, Andras (2003) "El papel del aseguramiento en la protección social. Revista de la CEPAL 81, diciembre 2003, p. 103.

- Tortuero Plaza, José Luis y del Aguila Cazorla, Olimpia (2004) "Los sistemas de pensiones en Europa y Latinoamérica: realidades, expectativas e ideas para un debate". Revista del Ministerio de Trabajo y Asuntos Sociales. Seguridad Social, N ${ }^{\circ}$ 54, p. 53.

- Toutain, S. (2007) Le nouvel âge des retraites, Ellipse.

- Uthoff, Andras (2006) «Brechas del Estado de bienestar y reformas de pensiones en América Latina»., Revista de la CEPAL 89, agosto, p. 9 .

- Valdés-Prieto, Salvador (2002) Políticas y mercados de pensiones. Un texto Universitario para América latina. Ediciones Universidad Católica de Chile, Santiago. 
- Valdés-Prieto, Salvador (1997) «La sécurité sociale dans les réformes des retraites en Amérique latine». Economie Internationale, $\mathrm{n}^{\circ} 72,4^{\circ}$ trimestre, p. 107.

- Walker E, Francisco y Cifuentes Lillo, Hugo. (2008) "Aspectos generales de la ley que establece una reforma previsional". Revista Laboral Chilena, Abril, pp. 88-127.

- Walker E., Francisco y Cifuentes Lillo, Hugo (2007) "Principales aspectos institucionales del Proyecto de Ley que perfecciona el Sistema de Pensiones". Revista de Economía y Administración, Facultad de Economía y Negocios, Universidad de Chile, Mayo, $\mathrm{N}^{\circ}$ 154, pp. 58-69.

- World BANK (2005) Old age income support in the 21st Century: An International Perspective on Pension Systems and Reform. World Bank.

- World Bank (1994) Averting the Old Age Crisis: Policies to Protect the Old and Promote Growth. World Bank, Oxford University Press. 